\title{
A comparison between the administration of oral prolonged-release oxycodone-naloxone and transdermal fentanyl in patients with moderate- to-severe cancer pain: a propensity score analysis
}

This article was published in the following Dove Press journal:

Journal of Pain Research

4 September 2017

Number of times this article has been viewed

\author{
A Roberto' \\ MT Greco ${ }^{2}$ \\ L Legramandi ${ }^{3}$ \\ F Galli ${ }^{3}$ \\ $M$ Galli ${ }^{4}$ \\ O Corli'
}

'Pain and Palliative Care Research Unit, Oncology Department, IRCCSIstituto di Ricerche Farmacologiche Mario Negri, Milan, Italy, ${ }^{2}$ Department of Clinical Sciences and Community, University of Milan, Milan,

Italy, ${ }^{3}$ Methodology for Clinical Research Laboratory, Oncology Department, IRCCS-Istituto di Ricerche Farmacologiche Mario Negri, Milan, Italy, ${ }^{4}$ Scientific Medical Communication srl, Novara, Italy
Correspondence: Maria Teresa Greco Department of Clinical Sciences and Community, University of Milan, Via Francesco Sforza 35-20, I 22 Milan, Italy $\mathrm{Tel}+390239014648$

Email mtgrc@yahoo.it
Background: Opioids are the most important pharmacological treatment for moderate-to-severe cancer pain, but side effects limit their use. Transdermal fentanyl (TDF) and oral prolongedrelease oxycodone-naloxone (OXN-PR) are effective in controlling chronic pain, with less constipation compared to other opioids. However, TDF and OXN-PR have never been directly compared.

Patients and methods: Cancer patients with moderate-to-severe chronic pain were consecutively enrolled in two prospective 28-day trials, received either TDF or OXN-PR, and were assessed at baseline and after 7, 14, 21, and 28 days. The primary endpoint was 28-day analgesic response rate (average pain intensity decrease $\geq 30 \%$ from baseline). Other outcome measures included opioid daily dose changes over time; need for adjuvant analgesics; number of switches; premature discontinuation; presence and severity of constipation; and other adverse drug reactions. To compare the efficacy and the safety of TDF and OXN-PR, we used the propensity score analysis to adjust for heterogeneity between the two patient groups.

Results: Three hundred ten out of 336 patients originally treated (119 TDF and 191 OXN-PR) were included in the comparative analysis. The amount of responders was comparable after TDF (75.3\%) and OXN-PR administration (82.9\%, not significant [NS]). The final opioid daily dose expressed as morphine equivalent was $113.6 \mathrm{mg}$ for TDF and $44.5 \mathrm{mg}$ for OXN-PR $(p<0.0001)$. A daily opioid dose escalation $>5 \%$ was less common after OXN-PR $(19.3 \%)$ than after TDS administration $(37.9 \%, p<0.0001)$. Opioid switches and discontinuation were similar in both groups. Severe constipation in the two groups was comparable (32.6\% after TDF vs $24.7 \%$ after OXN-PR, NS). Nausea, vomiting, and dry mouth were significantly less frequent in the OXN-PR group than in the TDF group.

Conclusion: Despite a similar analgesic activity in moderate-to-severe cancer pain, OXN-PR is characterized by lower daily dosages, less need for drug escalation, and fewer side effects compared to TDF.

Keywords: opioid induced constipation, oxycodone-naloxone, transdermal fentanyl, propensity score, analgesic efficacy, constipation

\section{Background}

The World Health Organization (WHO) ${ }^{1}$ and recent international guidelines for the pharmacological treatment of cancer pain suggest that opioids are the best established therapy for moderate-to-severe cancer pain, recommending oral morphine, oxycodone, and hydromorphone as primary options for strong opioid therapy (Step III)., Despite some variability in their responses, a recent study found comparable efficacy 
and tolerability profiles of morphine, oxycodone, and either transdermal fentanyl (TDF) or buprenorphine in cancer patients naïve to strong opioids. ${ }^{4}$

However, burdensome side effects limit the compliance to opioids in the long term, because they often induce bowel dysfunction, including constipation, incomplete evacuation, hard dry stools, bloating, abdominal cramping, and gastric reflux. ${ }^{5}$ The European Association of Palliative Care (EAPC) and the European Society of Medical Oncology strongly recommend routine laxative prescriptions for prophylaxis and management of opioid-induced bowel dysfunction in advanced cancer patients receiving opioids. ${ }^{2,6}$

To decrease the gastrointestinal adverse effects of opioids, oral prolonged-release oxycodone combined with naloxone (OXN-PR) in a 2:1 ratio was developed. ${ }^{7}$ The binding of oxycodone to the intestinal opioid receptors is strongly opposed by the opioid receptor antagonist naloxone, due to the higher receptor affinity of the latter. Conversely, the extensive hepatic first-pass metabolism limits the oral administered naloxone's bioavailability, thus avoiding the interference with the central analgesic action of oxycodone. ${ }^{7}$

In randomized controlled trials (RCTs) and postmarketing studies, OXN-PR has caused less opioid-induced bowel dysfunction than traditional opioids, particularly oral ones, with similar or even greater analgesic efficacy. ${ }^{8-10}$ Limited data, mainly from observational studies, are available on OXN-PR in cancer pain, ${ }^{11,12}$ and the efficacy and tolerability of OXN-PR and TDF have never been compared so far.

To partially cover this gap, we compared the analgesic efficacy and safety profiles of OXN-PR and TDF in strong opioid-naïve patients affected by moderate-to-severe chronic cancer pain. We used a propensity score (PS) analysis, a widely employed statistical method, ${ }^{13,14}$ to compare data recorded in nonrandomized treatment groups from different observational studies.

\section{Patients and methods}

\section{Study design, patients, and assessment}

Consecutive cancer patients with moderate-to-severe pain and naïve to WHO-Step III opioids were enrolled in two prospective 28-day multicenter studies (NCT01809106 ${ }^{15}$ and NCT02293785) and received either TDF or OXN-PR. The two trials were carried out sequentially in Italy in 2011-2015 and had identical clinical settings, eligibility criteria, efficacy and tolerability endpoints, and outcome measures.

Briefly, patients were included in the two studies if they met the following eligibility criteria: age $>18$ years; locally advanced or metastatic tumor (with the exclusion of cerebral tumors and leukemia given their different pain mechanisms); persistent moderate-to-severe cancer pain (average pain intensity [API] in the last $24 \mathrm{~h} \geq 4$ points on a $0-10$ numerical rating scale, where 0 indicates no pain and 10 the worst imaginable pain) requiring WHO-Step III opioids and suitable for treatment with OXN-PR or TDF; no previous treatment with WHO-Step III opioids (strong opioid naïve). Pregnant women, patients with preexisting renal failure, history of alcohol or drug abuse, cognitive impairment, and those who received chemo- or radiotherapy in the 7 days before the observation period or any nonpharmacological analgesics were excluded.

Patients were evaluated at baseline (T0) and at 7, 14, 21 , and 28 days thereafter, and they were closely monitored through a careful titration of their analgesic treatment. Demographic information (age and sex) and details of clinical history (primary tumor site, sites of metastases, previous treatments for pain, previous and ongoing cancer treatments, concomitant diseases, and Karnofsky Performance Status) were recorded at $\mathrm{T} 0$. The following pain characteristics were assessed: API experienced in the previous $24 \mathrm{~h}$; neuropathic pain, evaluated by the Douleur Neuropathique 4 [Neuropathic Pain 4] inventory, ${ }^{16}$ which consists of pain descriptors and bedside sensory examinations commonly used in the clinical practice and research; type of pain (nociceptive, neuropathic, mixed); and breakthrough pain (BTP) according to the Davies algorithm. ${ }^{17}$ Overall therapy under way at the beginning of the study and any new drugs scheduled during observation were also recorded.

The starting dose of TDF $(12.5-25 \mu \mathrm{g} / \mathrm{h})$ or OXN-PR (5/2.5-20/10 mg/day) was determined by a pain physician according to the EAPC recommendations ${ }^{2}$ and by considering each patient's needs and previous analgesic therapy. During follow-up, physicians were allowed to adjust the starting dose to better control the pain. The adjustments could include not only changes in the dose but also addition of another opioid or adjuvant $\operatorname{drug}(\mathrm{s})$, either discontinuation of the original therapeutic strategy or switching to another strong opioid in case of unsatisfactory analgesia, or intolerable adverse drug reactions (ADRs).

\section{Outcome measures}

During each visit, the analgesic efficacy was determined on the basis of the API. Other measures included around-theclock (ATC) opioid daily doses (expressed as oral morphineequivalent daily dose, mg); changes of analgesic therapy, type(s) and dose(s) of extra opioids or adjuvant drugs; opioid switch or discontinuation. 
Safety was assessed at any visit using a self-administered questionnaire to record any side effect that appeared or changed in intensity or frequency after the TDF or OXN-PR administration. ADR severity was measured by means of a four-point verbal rating scale (no, light, moderate, severe), according to the Therapy Impact Questionnaire. ${ }^{18}$

Based on the existing data on the clinically meaningful changes of this parameter, ${ }^{4,19,20}$ we considered as "Responders" those patients who obtained an average pain intensity difference (APID) $\geq 30 \%$ by comparing the first and last visit. The proportion of responders (for both TDF and OXN-PR) was considered as the primary efficacy endpoint.

To evaluate the overall risk-benefit profile of TDF and OXN-PR, an additional co-primary composite efficacy and tolerability endpoint (clinical success) was used and it included the simultaneous presence of APID $\geq 50 \%$ and absence of moderate or severe ADRs in the study period, indicating a worthwhile analgesic benefit.

The secondary endpoints included 1) opioid dose increases after the observation time; 2) the proportion of patients requiring a mean increase of $>5 \%$ in the opioid daily dose according to the Opioid Escalation Index\% (OEI\%), ${ }^{21}$ defined as follows:

$$
\mathrm{OEI} \%=\frac{\frac{(\text { Final opioid daily dose }- \text { Opioid daily dose } \mathrm{T} 0)}{\text { Opioid daily dose } \mathrm{T} 0}}{\text { Number of days }} * 100
$$

3) the proportion of patients requiring a switch to another opioid because of inefficacy or toxicity; 4) the proportion of patients needing supplementary doses of opioids or adjuvant analgesic drugs to optimize the ATC therapy; 5) the proportion of patients discontinuing the opioid for pain treatment-related reasons; and 6) the proportion of patients with severe constipation.

\section{Statistical analysis}

In the descriptive analyses, absolute frequency was used for categorical variables, and central trend and dispersion measurements (mean, standard deviations) were used for quantitative continuous variables. Continuous/ordinal variables were checked for normality of data distribution by using Shapiro-Wilk test and were then compared by running either one-way analysis of variance or nonparametric tests. Chi-square or Fisher's exact tests were used for categorical variables. Linear interpolation was used to impute intermittent missing scores; the last observation carried forward was used to impute missing values in the event of early discontinuation.
To compare the efficacy and safety of TDF and OXNPR, we used a PS analysis to adjust for differences between the two patient groups. PS aims to allocate each patient the conditional probability of receiving one of the treatments available in an observational study, with a function of confounding variables that influence the choice of treatment by a physician. ${ }^{22,23}$ The probability of being assigned to TDF or OXN-PR was determined using a multivariable logistic regression model that included the following explanatory variables: age, sex, metastasis, API, Karnofsky Performance Status, previous anticancer therapy, previous WHO-II therapy, and constipation at baseline.

The PS score was also included in the generalized linear outcome model as a covariate to allow the comparison between the two samples.

A $p$-value of $<0.05$ was considered as statistically significant. All statistical analyses were performed with SAS 9.2 statistical software (SAS Institute, Cary, NC, USA).

\section{Ethics approval and consent to participate}

For both studies and in each participating center, the study protocol obtained the ethical approval and patients provided a written informed consent before any study-related activities were carried out (for details see Supplementary material).

\section{Results}

Three hundred thirty-six patients were originally enrolled in the two studies and treated for moderate-to-severe cancer pain with either TDF or OXN-PR. However, only 310 patients (119 TDF and 191 OXN-PR) were included in the final comparative analysis by PS (Figure 1).

Table 1 shows the baseline clinical characteristics according to the different treatments. The most common sites of cancer were the respiratory, digestive, and genitourinary systems. Over $80 \%$ of patients in each group reported metastases at baseline and over $70 \%$ received anticancer therapy. Almost three quarters of the TDF patients $(70.5 \%)$ and less than half of the OXN-PR patients $(47.1 \% ; p=0.0016)$ switched from a weak opioid to a new analgesic. The API at entry was 6.2 in both groups, with a high prevalence of nociceptive pain $(>80 \%)$ compared to neuropathic or mixed pain. Almost a half of the patients reported episodes of BTP at entry.

The results about study adherence are reported in Table 2 : over the 28-day follow-up, $52(16.8 \%)$ patients prematurely either withdrew from the treatment or were withdrawn from the observation, and 24 (7.7\%) patients were lost at follow-up. 


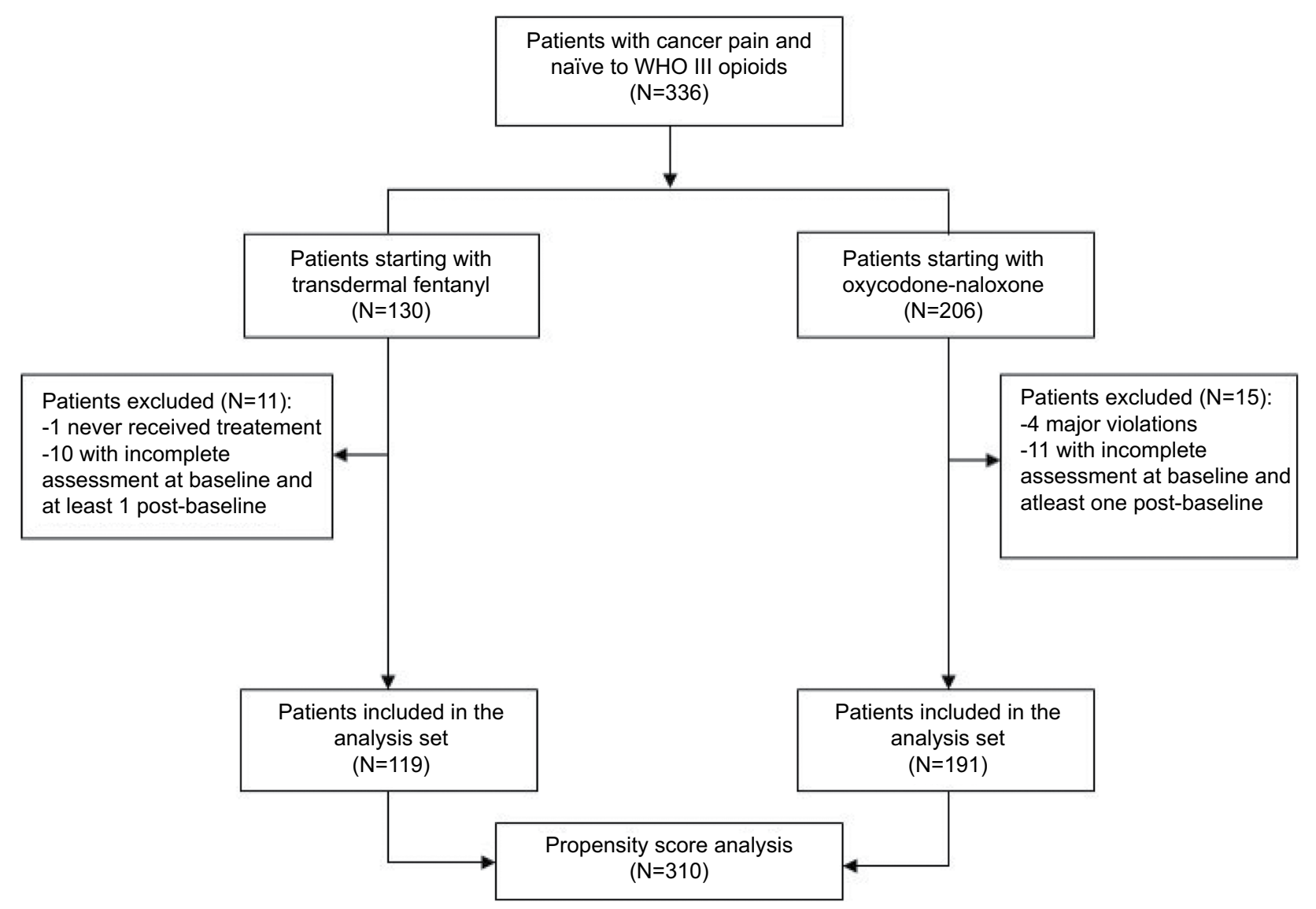

Figure I Study flowchart.

Abbreviation: WHO, World Health Organization.

The remaining 234 (75.4\%) patients continued with the same analgesic treatment up to the end of the observation.

\section{Analgesic efficacy}

Clinical measures and endpoints are reported in Table 3. The proportions of responders were not significantly different after the administration of either TDF or OXN-PR (75.3\% and $82.9 \%$, respectively; $p=0.11$ ). In both groups, API decreased from baseline to day 28 (Figure 2): from $6.2 \pm 1.5$ to $2.4 \pm 2.0$ in patients administered with TDF and from $6.2 \pm 1.1$ to $2.6 \pm 1.7$ in patients administered with OXN-PR (difference not significant).

Significant differences in opioid daily doses were recorded between the two groups at baseline (TDF: 53.0 mg; OXN-PR: $25.4 \mathrm{mg} ; p<0.001)$. This difference increased during the observation (Figure 3 ): after 28 days, the opioid daily dose was $113.6 \mathrm{mg}$ in patients treated with TDF, and $44.5 \mathrm{mg}$ in patients treated with OXN-PR $(p<0.0001)$. Thus, an opioid daily dose escalation $>5 \%$ was less common after OXN-PR (19.3\% vs 37.9\% after TDS, $p<0.0001)$ compared to TDF. At the end of the study, the rates of clinical success after TDF and OXN-PR were not significantly different (27.8\% and $36.5 \%$, respectively; $p=0.10$ ).

No significant differences were found between TDF and OXN-PR regarding the proportions of patients who required either adjuvant drugs or additional opioids, or switched to other analgesics during the study period (Table 3 ).

\section{Tolerability and safety}

The types and severity of the ADRs recorded during the study in TDF and OXN-PR patients are reported in Table 4. Nausea, vomiting, and dry mouth were more common in TDF patients compared to OXN-PR ones. Severe constipation was comparable in the two groups $(32.6 \%$ after TDF administration vs $24.7 \%$ after OXN-PR administration, not significant [NS]).

\section{Discussion}

This is the first study comparing the efficacy and safety of TDF and OXN-PR in cancer patients with moderate-to-severe pain. TDF is a strong opioid usually administered to patients with stable opioid requirements and suggested as an effective alternative to oral opioids for some patients (i.e., those unable 
Table I Main baseline demographic and clinical characteristics of the study population

\begin{tabular}{|c|c|c|}
\hline Main characteristics & $\begin{array}{l}\text { Transdermal } \\
\text { fentanyl } \\
(\mathbf{N}=\text { II9) }\end{array}$ & $\begin{array}{l}\text { PR oxycodone- } \\
\text { naloxone } \\
(\mathrm{N}=191)\end{array}$ \\
\hline Age (years) & $67.8(10.7)$ & $68.7(9.8)$ \\
\hline Female & $53(44.5 \%)$ & $83(43.5 \%)$ \\
\hline \multicolumn{3}{|l|}{ Primary site of tumor } \\
\hline Respiratory system & $34(28.6 \%)$ & 76 (39.8\%) \\
\hline Digestive system & $27(22.7 \%)$ & $50(26.2 \%)$ \\
\hline Genitourinary system & $24(20.2 \%)$ & $32(16.8 \%)$ \\
\hline Breast & $10(8.4 \%)$ & $10(5.2 \%)$ \\
\hline Head, neck & $12(10.1 \%)$ & $10(5.2 \%)$ \\
\hline Others & $12(10.1 \%)$ & $13(6.8 \%)$ \\
\hline Metastases & 99 (83.2\%) & $160(83.8 \%)$ \\
\hline Previous anticancer therapy & 91 (76.5\%) & 134 (70.2\%) \\
\hline Surgery & $55(60.4 \%)$ & $70(52.2 \%)$ \\
\hline Chemotherapy & $70(76.9 \%)$ & 107 (79.9\%) \\
\hline Biologic medical products & $17(18.7 \%)$ & $21(15.7 \%)$ \\
\hline Hormone therapy & $12(13.2 \%)$ & $17(12.7 \%)$ \\
\hline Radiotherapy & 35 (38.5\%) & 48 (35.8\%) \\
\hline Other & $4(4.4 \%)$ & $7(5.2 \%)$ \\
\hline Karnofsky Performance Status & $67.4(17.3)$ & $66.3(16.5)$ \\
\hline \multicolumn{3}{|l|}{ Previous pain therapy } \\
\hline No therapy (WHO-Step 0) & $13(11.1 \%)$ & $26(13.6 \%)$ \\
\hline Non-opioids (WHO-Step I) & $21(17.8 \%)$ & 75 (39.3\%) \\
\hline Weak opioids (WHO-Step II) & $84(70.5 \%)$ & 90 (47.1\%) \\
\hline Adjuvant pain therapies & $54(45.4 \%)$ & $92(48.2 \%)$ \\
\hline Steroids & $32(56.3 \%)$ & $64(69.6 \%)$ \\
\hline Anticonvulsants & $9(16.7 \%)$ & $16(17.4 \%)$ \\
\hline Antidepressants & $6(11.1 \%)$ & $14(15.2 \%)$ \\
\hline Bisphosphonates & $7(13.0 \%)$ & $10(10.9 \%)$ \\
\hline Other & $8(14.8 \%)$ & $10(10.9 \%)$ \\
\hline Pain duration (months) & $3.2(4.5)$ & $3.3(3.7)$ \\
\hline Average pain intensity, NRS & $6.2(1.5)$ & $6.2(1.1)$ \\
\hline \multicolumn{3}{|l|}{ Type of pain } \\
\hline Only nociceptive & $102(85.7 \%)$ & 155 (8I.2\%) \\
\hline Only neuropathic & - & - \\
\hline Mixed & $17(14.3 \%)$ & $36(18.8 \%)$ \\
\hline Breakthrough pain & 55 (46.2\%) & $82(42.9 \%)$ \\
\hline Constipation & $31(26.1 \%)$ & $74(38.7 \%)$ \\
\hline
\end{tabular}

Note: Values are mean ( \pm standard deviation) or number of patients (\%). Abbreviations: NRS, numerical rating scale; PR, prolonged release; WHO, World Health Organization. to swallow), according to international recommendations. ${ }^{2,6}$ By combining the opioid agonist-antagonist oxycodone and naloxone, OXN-PR is aimed at relieving pain and limiting opioid-induced constipation.

In the absence of interventional studies, these analyses allow comparing the analgesic efficacy and safety of TDF and OXN-PR. We analyzed data collected in two studies recently performed by our group and aimed at evaluating the characteristics of different opioids commonly used in cancer patients. To reduce the effects of confounding factors and to account for systematic differences in baseline characteristics between TDF and OXN-PR subjects, we applied the PS, an alternative statistical method previously used to compare the analgesic effectiveness between opioids in cancer patients. ${ }^{12,13}$

In 28 days of treatment, pain intensity decreased about $60 \%$ in both groups. A previous RCT showed that TDF and oxycodone (not in combination with naloxone) had equivalent analgesic effects in chronic cancer pain. ${ }^{15}$ The present analysis indicates that OXN-PR achieves comparable analgesic efficacy of TDF. Additionally, as previously mentioned, we considered as responders the patients who achieved a decrease of $30 \%$ or more of pain intensity from baseline to final visit, based on the Farrar criterion. ${ }^{19,24-27}$ Although the difference was not significant, by testing the primary efficacy endpoint of this analysis we found that the proportion of responders was slightly higher after OXN-PR compared to TDF administration.

The final to basal pain intensity difference and the positive response rate provided different information on the analgesic effect. In clinical studies, the results are mainly driven by the whole population's mean values for specified outcomes and end points. The former method offers a general picture of the efficacy of a given treatment but does not allow to distinguish good from bad responses. A poor analgesic effect is a primary issue in clinical practice and a lack of response is

Table 2 Study adherence

\begin{tabular}{lll}
\hline & Transdermal fentanyl (N=I I9), $\mathbf{n}(\%)$ & PR oxycodone-naloxone (N= I 9 I), $\mathbf{n}(\%)$ \\
\hline Completed the 28-day study & $87(73.1)$ & I $47(76.9)$ \\
$\begin{array}{l}\text { Premature treatment discontinuation } \\
\text { Reasons for discontinuation }\end{array}$ & $27(22.6)$ & $25(13.1)$ \\
$\quad$ & \\
Noncompliance & $\mathrm{I}(3.7)$ & $3(12.0)$ \\
Refusal & $0(0)$ & $\mathrm{I}(4.0)$ \\
Death & $10(37.0)$ & $\mathrm{I}(4.0)$ \\
Other & $4(14.8)$ & $2(8.0)$ \\
Inadequate analgesia & $8(29.6)$ & $7(28.0)$ \\
Severe ADRs & $4(14.8)$ & $4(16.0)$ \\
Inadequate analgesia and severe ADR & $0(0)$ & $\mathrm{I}(4.0)$ \\
Difficulty swallowing & $0(0.0)$ & $6(24.0)$ \\
Lost to follow-up & $5(4.2)$ & $19(9.9)$ \\
\hline
\end{tabular}

Abbreviations: ADRs, adverse drug reactions; PR, prolonged release. 
Table 3 Clinical measures and end points

\begin{tabular}{|c|c|c|c|}
\hline & $\begin{array}{l}\text { Transdermal fentanyl } \\
(\mathrm{N}=\text { II9) }\end{array}$ & $\begin{array}{l}\text { Oral oxycodone-naloxone } \\
(N=191)\end{array}$ & $p$-value \\
\hline Responders & $75.3 \%(3.7)$ & $82.9 \%(2.9)$ & 0.11 \\
\hline Clinical success & $27.8 \%(4.4)$ & $36.5 \%(3.4)$ & 0.12 \\
\hline Starting opioid dose $(\mathrm{mg} / \text { day })^{\mathrm{a}}$ & $53.0(1.3)$ & $25.4(1.0)$ & $<0.0001$ \\
\hline Final dose $(\mathrm{mg} / \text { day })^{\mathrm{a}}$ & II $3.6(4.9)$ & $44.5(3.9)$ & $<0.0001$ \\
\hline Mean dose increase ${ }^{a}$ & $2.5(1.8)$ & $0.4(1.4)$ & $<0.0001$ \\
\hline OEI $>5 \%^{a}$ & $37.9 \%(4.1)$ & $19.3 \%(3.2)$ & $<0.0001$ \\
\hline Patients requiring additional opioids & $37.1 \%(4.4)$ & $28.2 \%(3.4)$ & 0.12 \\
\hline Patients requiring adjuvant drugs & $83.7 \%(3.5)$ & $82.4 \%(2.8)$ & 0.78 \\
\hline Switches & $14.1 \%(3.1)$ & $10.6 \%(2.4)$ & 0.37 \\
\hline Premature discontinuations due to ADRs & $2.8 \%(1.2)$ & $0.9 \%(0.9)$ & 0.22 \\
\hline Severe constipation & $32.6 \%(4.2)$ & $24.7 \%(3.3)$ & 0.15 \\
\hline
\end{tabular}

Notes: Values are percentage or number (standard error); ${ }^{\text {a }}$ around-the-clock daily doses (as oral morphine-equivalent daily dose).

Abbreviations: ADRs, adverse drug reactions; OEI, opioid escalation index.

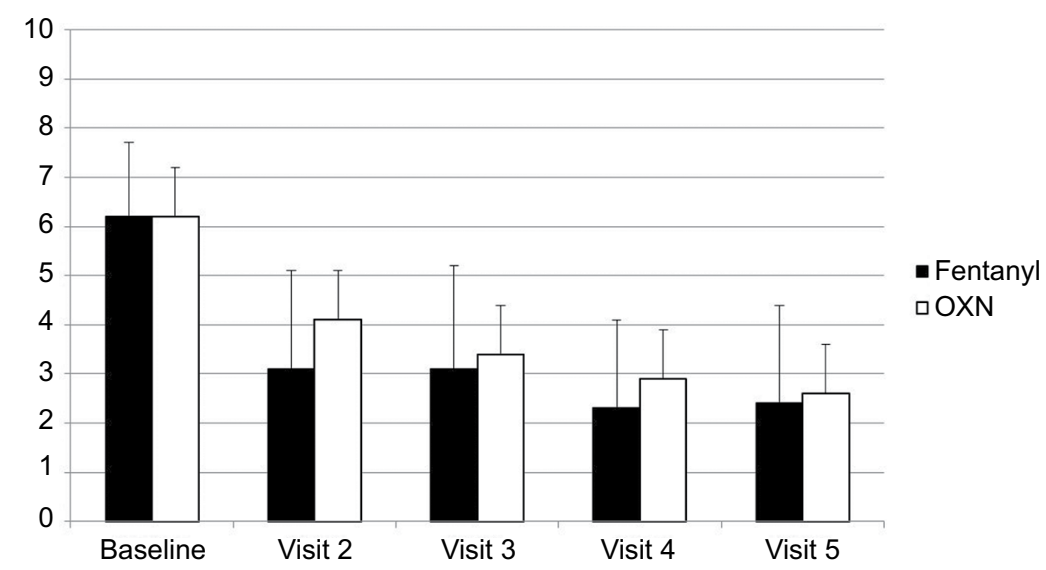

Figure 2 Mean pain intensity score (II-point numerical rating scale) throughout observation after transdermal fentanyl and prolonged-release oxycodone-naloxone. Abbreviation: OXN, oxycodone-naloxone.

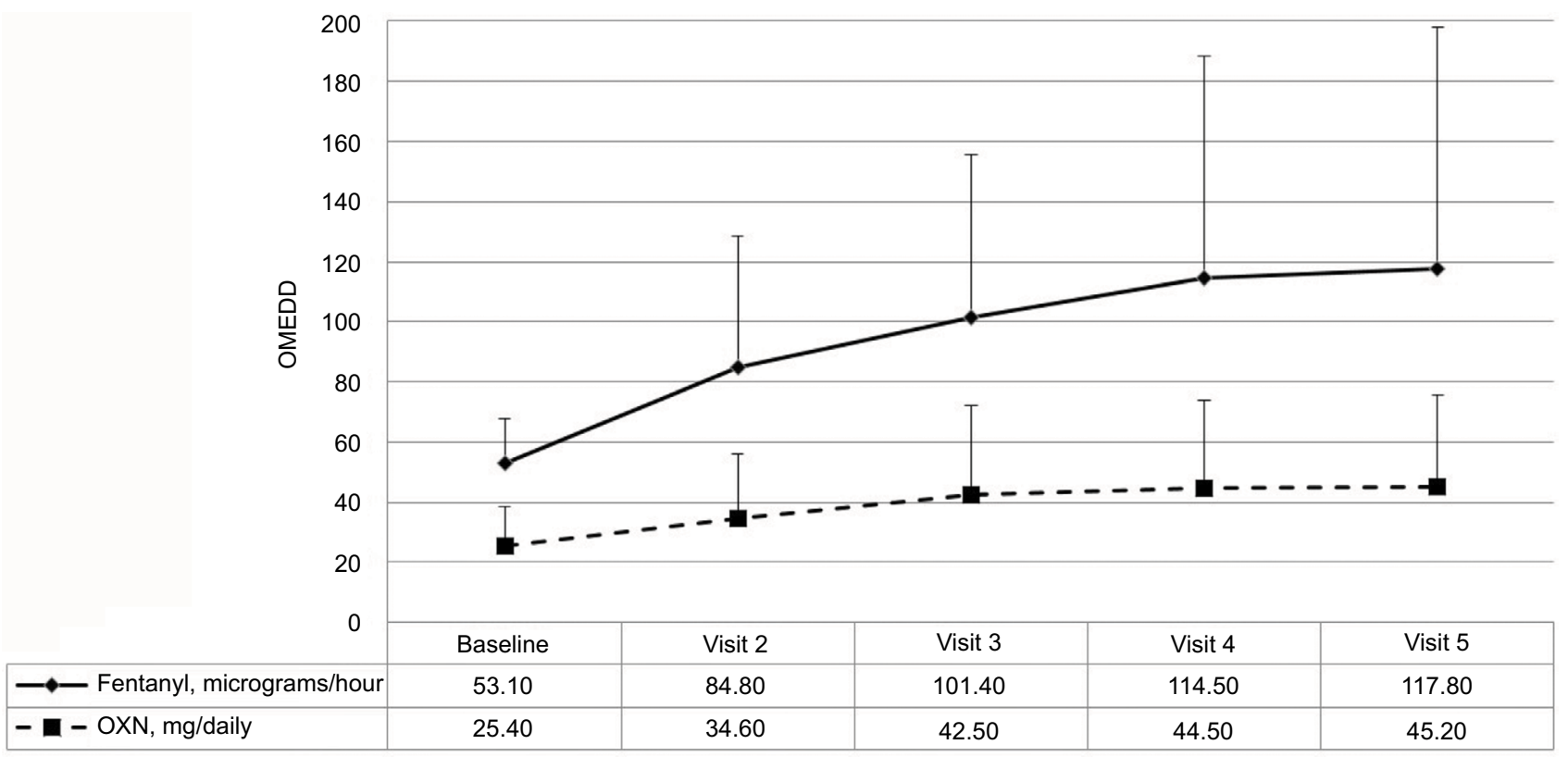

Figure 3 Transdermal fentanyl and prolonged-release oxycodone-naloxone daily dosages (expressed in oral morphine-equivalent daily dose). Abbreviations: OMEDD, oral morphine-equivalent daily dose; OXN, oxycodone-naloxone. 
Table 4 Patients with adverse drug reactions in each treatment arm

\begin{tabular}{|c|c|c|c|}
\hline & $\begin{array}{l}\text { Transdermal } \\
\text { fentanyl } \\
(\mathbf{N}=1 \mid 9)\end{array}$ & $\begin{array}{l}\text { Oxycodone- } \\
\text { naloxone } \\
(\mathbf{N}=191)\end{array}$ & $p$-value \\
\hline \multicolumn{4}{|l|}{ Drowsiness } \\
\hline Any degree & $68(57.1)$ & $113(59.2)$ & 0.72 \\
\hline Severe & $24(20.2)$ & 37 (19.4) & 0.86 \\
\hline \multicolumn{4}{|l|}{ Confusion } \\
\hline Any degree & $45(37.8)$ & $69(36.1)$ & 0.76 \\
\hline Severe & $8(6.7)$ & $17(8.9)$ & 0.49 \\
\hline \multicolumn{4}{|l|}{ Nausea } \\
\hline Any degree & $55(46.2)$ & 64 (33.5) & 0.02 \\
\hline Severe & $15(12.6)$ & $17(8.9)$ & 0.29 \\
\hline \multicolumn{4}{|l|}{ Vomiting } \\
\hline Any degree & $28(23.5)$ & $28(14.7)$ & 0.05 \\
\hline Severe & $10(8.4)$ & II (5.8) & 0.36 \\
\hline \multicolumn{4}{|l|}{ Constipation } \\
\hline Any degree & $76(63.9)$ & $115(60.2)$ & 0.51 \\
\hline Severe & $36(30.2)$ & $50(26.2)$ & 0.43 \\
\hline \multicolumn{4}{|l|}{ Dry mouth } \\
\hline Any degree & $66(55.5)$ & 79 (4I.4) & 0.02 \\
\hline Severe & $29(24.4)$ & $23(12.0)$ & 0.01 \\
\hline \multicolumn{4}{|c|}{ Hallucinations } \\
\hline Any degree & $3(2.5)$ & $14(7.3)$ & 0.07 \\
\hline Severe & - & $2(1.1)$ & 0.26 \\
\hline \multicolumn{4}{|c|}{ Muscle spasm/myoclonus } \\
\hline Any degree & $15(12.6)$ & $27(14.1)$ & 0.70 \\
\hline Severe & $5(4.2)$ & $3(1.6)$ & 0.26 \\
\hline \multicolumn{4}{|l|}{ Gastralgia } \\
\hline Any degree & $26(21.8)$ & $42(22.0)$ & 0.97 \\
\hline Severe & $4(3.4)$ & $8(4.2)$ & 0.22 \\
\hline \multicolumn{4}{|l|}{ Dysuria } \\
\hline Any degree & $13(10.9)$ & $19(10.0)$ & 0.78 \\
\hline Severe & $4(3.4)$ & $5(2.6)$ & 0.24 \\
\hline \multicolumn{4}{|c|}{ Breathlessness } \\
\hline Any degree & $21(17.6)$ & $23(12.0)$ & 0.16 \\
\hline Severe & $4(3.4)$ & $4(2.1)$ & 0.22 \\
\hline \multicolumn{4}{|l|}{ Itching } \\
\hline Any degree & $13(10.9)$ & $17(8.9)$ & 0.55 \\
\hline Severe & $3(2.5)$ & $3(1.6)$ & 0.26 \\
\hline
\end{tabular}

Note: Data presented as $n$ (\%).

a problem that needs to be understood and solved. Patients reporting pain intensity reduction $<30 \%$ were considered nonresponders. They amounted to $24.7 \%$ and $17.1 \%$ in the TDF and OXN-PR groups, respectively.

These data confirm the results of a recent RCT study aimed at comparing both efficacy and safety of four strong opioids in cancer patients. ${ }^{15}$ They found that nonresponders ranged from $20 \%$ to $25 \%$ and that this variability depended on the administered drug. Noteworthy, nonresponders are by definition poorly susceptible to the action of opioids and this condition leads to a compensating increase of dose over time.

We observed a higher starting dose and a greater dose increase across the observation time in patients treated with
TDF. The first might depend on frequent previous therapies with weak opioids in this group that quite likely induced higher doses requirement when starting the WHO-Step III. Independently from these basal conditions, we found an increase of $120 \%$ in the doses administered to the TDF group while the increase of the doses in the OXN-PR group reached $80 \%$. This difference was confirmed by the $\mathrm{OEI} \%>5$, significantly higher in TDF compared to OXNPR patients.

Different degrees of dose escalation over time can generally be attributed to dissimilar tendencies to develop tolerance. Even if a comparative clinical study on the onset of tolerance to different opioids has not yet been performed, the degrees of tolerance for TDF and oxycodone have already been reported. ${ }^{15}$

The prevalence of ADRs was substantially comparable after TDF and OXN-PR, except for nausea, vomiting, and dry mouth, which were more frequent among TDF recipients. A lower prevalence of nausea and vomiting after OXN-PR was also reported in other studies ${ }^{12,28}$ and a possible explanation might be that the opioid antagonist naloxone acts at the gastric level through the same mechanism as in limiting bowel dysfunction, thus reducing the development of nausea and vomiting. Constipation is a frequent problem, occurring in more than $40 \%$ of the patients treated with opioids..$^{29} \mathrm{In}$ previous studies, ${ }^{30,31}$ TDF was associated with less constipation than oral opioids. In the present analysis, the number of patients complaining of constipation was similar between TDF and OXN-PR.

Despite the notable aspects being addressed by the present analysis, we acknowledge that this study has several inherent limitations because of the method used to control for the imbalance between comparison groups. The assumption that high variance was considered in the model used to estimate the PS should be properly demonstrated. To optimize the PS, variables were selected by considering evidences in the literature ${ }^{8,32}$ and expert recommendations. ${ }^{22,33}$ Unlike randomization, PS analysis can only remove overt (known) bias, while hidden (unmeasured) biases remain. Therefore, the results generated using PS analysis must be carefully interpreted. The validity of the results depends on the quality and the quantity of information about the efficacy and safety of the evaluated treatments. The sample population characteristics are also important: PS analysis allows partial extrapolations to different patient groups and settings, with different distribution in the covariates used to generate the score. Different considerations arose from these results. First, by acknowledging the limits of the PS analysis, the problem of the generalizability of the results (external validity) becomes central; 
second, there is a clear need to carefully take into account additional important determinants, which are involved in the physicians' and patients' decisions about whether or not to prescribe and use a treatment. However, despite its inherent limitations, the PS model may improve the understandings of the real value of results from observational studies. To date, only one RCT has compared the efficacy and safety of oxycodone-PR and OXN-PR in cancer pain, by involving an extremely selected cohort of patients with controlled pain and opioid-induced constipation at baseline. ${ }^{34}$ Future efforts should be aimed at comparing opioids in unselected patients with cancer by taking advantage of RCTs.

\section{Conclusion}

We analyzed patients affected by moderate-to-severe cancer pain and treated with either TDF or OXN-PR. Our results showed that the two opioids induced comparable analgesic efficacy, despite markedly lower daily dosages and less need for drug escalation in case of OXN-PR administration, thus highlighting less mid-term opioid tolerance with the agonist-antagonist combination. Nausea and vomiting were also reduced after OXN-PR, while constipation was comparable for the two opioids.

\section{Acknowledgments}

This study was completed independently, and the authors were the sole persons involved in the study design, data collection, and analysis, decision to publish, and preparation of the manuscript.

\section{Disclosure}

Dr. M. Galli was the scientific director of Mundipharma Pharmaceuticals until January 2017. Other authors report no conflicts of interest in this work.

\section{References}

1. World Health Organization. Cancer Pain Relief. 2nd ed. Geneva: World Health Organization; 1996. Available from http://apps.who.int/iris/ bitstream/10665/37896/1/9241544821.pdf. Accessed July 26, 2017.

2. Caraceni A, Hanks G, Kaasa S, et al; European Palliative Care Research Collaborative (EPCRC); European Association for Palliative Care (EAPC). Use of opioid analgesics in the treatment of cancer pain: evidence-based recommendations from the EAPC. Lancet Oncol. 2012;13(2):e58-e68.

3. Wiffen PJ. Evidence-based pain management and palliative care in issue one for 2005 of The Cochrane Library. J Pain Palliat Care Pharmacother. 2005;19(3):65-68.

4. Corli O, Montanari M, Deandrea S, Greco MT, Villani W, Apolone G. An exploratory analysis on the effectiveness of four strong opioids in patients with cancer pain. Pain Med. 2012;13(7):897-907.

5. Mancini I, Bruera E. Constipation in advanced cancer patients. Support Care Cancer. 1998;6(4):356-364.
6. Ripamonti CI, Santini D, Maranzano E, Berti M, Roila F. ESMO Guidelines Working Group. Management of cancer pain: ESMO Clinical Practice Guidelines. Ann Oncol. 2012;23 (Suppl 7):vii139-vii154.

7. Mundipharma Pharmaceuticals Limited. Targin $5 / 2.5 \mathrm{mg}, 10 \mathrm{mg} / 5 \mathrm{mg}$, $20 \mathrm{mg} / 10 \mathrm{mg}$ and $40 / 20 \mathrm{mg}$ prolonged release tablets: summary of Product Characteristics; 2013. Available from: http://www.medicines. ie/medicine/14383/SPC/Targin+10mg+5mg+and+20mg+10mg+prolo nged+release+tablets/. Accessed February 8, 2017.

8. Burness CB, Keating GM. Oxycodone-naloxone prolonged-release: a review of its use in the management of chronic pain while counteracting opioid-induced constipation. Drugs. 2014;74(3):353-375.

9. Koopmans G, Simpson K, De Andres J, Lux EA, Wagemans M, Van Megen Y. Fixed ratio (2:1) prolonged-release oxycodone-naloxone combination improves bowel function in patients with moderate-tosevere pain and opioid-induced constipation refractory to at least two classes of laxatives. Curr Med Res Opin. 2014;30(11):2389-2396.

10. Poelaert J, Koopmans-Klein G, Dioh A, et al. Treatment with prolongedrelease oxycodone-naloxone improves pain relief and opioid-induced constipation compared with prolonged-release oxycodone in patients with chronic severe pain and laxative-refractory constipation. Clin Ther. 2015;37(4):784-792.

11. Cuomo A, Russo G, Esposito G, Forte CA, Connola M, Marcassa C. Efficacy and gastrointestinal tolerability of oral oxycodone-naloxone combination for chronic pain in outpatients with cancer: an observational study. Am J Hosp Palliat Care. 2014;31(8):867-876.

12. Lazzari M, Greco MT, Marcassa C, Finocchi S, Caldarulo C, Corli O. Efficacy and tolerability of oral oxycodone and oxycodone/naloxone combination in opioid-naive cancer patients: a propensity analysis. Drug Des Devel Ther. 2015;9:5863-5872.

13. Apolone G, Deandrea S, Montanari M, Corli O, Greco MT, Cavuto S. Evaluation of the comparative analgesic effectiveness of transdermal and oral opioids in cancer patients: a propensity score analysis. Eur $J$ Pain. 2012;16(2):229-238.

14. D'Agostino RB Jr. Propensity score methods for bias reduction in the comparison of a treatment to a non-randomized control group. Stat Med. 1998;17(19):2265-2281.

15. Corli O, Floriani I, Roberto A, et al; CERP Study OF Pain Group (List of collaborators); CERP Study of Pain Group. Are strong opioids equally effective and safe in the treatment of chronic cancer pain? A multicenter randomized phase IV 'real life' trial on the variability of response to opioids. Ann Oncol. 2016;27(6):1107-1115.

16. Bouhassira D, Attal N, Alchaar H, et al. Comparison of pain syndromes associated with nervous or somatic lesions and development of a new neuropathic pain diagnostic questionnaire (DN4). Pain. 2005;114(1-2):29-36.

17. Davies AN, Dickman A, Reid C, Stevens AM, Zeppetella G; Science Committee of the Association for Palliative Medicine of Great Britain and Ireland. The management of cancer-related breakthrough pain: recommendations of a task group of the Science Committee of the Association for Palliative Medicine of Great Britain and Ireland. Eur J Pain. 2009;13(4):331-338.

18. Tamburini M, Rosso S, Gamba A, Mencaglia E, De Conno F, Ventafridda $\mathrm{V}$. A therapy impact questionnaire for quality-of-life assessment in advanced cancer research. Ann Oncol. 1992;3(7):565-570.

19. Corli O, Montanari M, Greco MT, et al. How to evaluate the effect of pain treatments in cancer patients: results from a longitudinal outcomes and endpoint Italian cohort study. Eur J Pain. 2013;17(6):858-866.

20. Kaasa S, Apolone G, Klepstad P, et al; European Palliative Care Research Collaborative (EPCRC); European Association for Palliative Care Research Network (EAPCRN). Expert conference on cancer pain assessment and classification-the need for international consensus: working proposals on international standards. BMJ Support Palliat Care. 2011;1(3):281-287.

21. Mercadante S, Fulfaro F, Casuccio A, Barresi L. Investigation of an opioid response categorization in advanced cancer patients. $J$ Pain Symptom Manage. 1999;18(5):347-352. 
22. Cavuto S, Bravi F, Grassi MC, Apolone G. Propensity score for the analysis of observational data: an introduction and an illustrative example. Drug Dev Res. 2006;67(3):208-216.

23. Rosenbaum PR. Rubin DB. The central role of the propensity score in observational studies for causal effects. Biometrika. 1983;70(1):41-55.

24. Dworkin RH, Turk DC, Wyrwich KW, et al. Interpreting the clinical importance of treatment outcomes in chronic pain clinical trials: IMMPACT recommendations. J Pain. 2008;9(2):105-121.

25. Farrar JT, Portenoy RK, Berlin JA, Kinman JL, Strom BL. Defining the clinically important difference in pain outcome measures. Pain. 2000;88(3):287-294

26. Farrar JT, Pritchett YL, Robinson M, Prakash A, Chappell A. The clinical importance of changes in the 0 to 10 numeric rating scale for worst, least, and average pain intensity: analyses of data from clinical trials of duloxetine in pain disorders. J Pain. 2010;11(2):109-118.

27. Farrar JT, Young JP Jr, LaMoreaux L, Werth JL, Poole RM. Clinical importance of changes in chronic pain intensity measured on an 11-point numerical pain rating scale. Pain. 2001;94(2):149-158.

28. Schutter U, Grunert S, Meyer C, Schmidt T, Nolte T. Innovative pain therapy with a fixed combination of prolonged-release oxycodone/ naloxone: a large observational study under conditions of daily practice. Curr Med Res Opin. 2010;26(6):1377-1387.
29. Benyamin R, Trescot AM, Datta S, et al. Opioid complications and side effects. Pain Physician. 2008;11 (Suppl 2):S105-S120.

30. Clark AJ, Ahmedzai SH, Allan LG, et al. Efficacy and safety of transdermal fentanyl and sustained-release oral morphine in patients with cancer and chronic non-cancer pain. Curr Med Res Opin. 2004;20(9):1419-1428.

31. Tassinari D, Sartori S, Tamburini E, et al. Transdermal fentanyl as a front-line approach to moderate-severe pain: a meta-analysis of randomized clinical trials. J Palliat Care. 2009;25(3):172-180.

32. Wiffen PJ, Derry S, Moore RA. Impact of morphine, fentanyl, oxycodone or codeine on patient consciousness, appetite and thirst when used to treat cancer pain. Cochrane Database Syst Rev. 2014;(5): CD011056.

33. Brookhart MA, Schneeweiss S, Rothman KJ, Glynn RJ, Avorn J, Sturmer T. Variable selection for propensity score models. Am J Epidemiol. 2006;163(12):1149-1156.

34. Ahmedzai SH, Nauck F, Bar-Sela G, Bosse B, Leyendecker P, Hopp M. A randomized, double-blind, active-controlled, double-dummy, parallel-group study to determine the safety and efficacy of oxycodone/ naloxone prolonged-release tablets in patients with moderate/severe, chronic cancer pain. Palliat Med. 2012;26(1):50-60. 


\section{Supplementary material}

\section{List of ethics committees for each participating center in both studies}

\section{CERP study (NCT01809106) - 44 centers}

1. Comitato Etico Per La Sperimentazione Clinica Dei Farmaci Della Ausl Di Pescara - Pescara

2. Comitato Etico Dell'universita' Degli Studi Gabriele D'annunzio E Della Asl 2 Lancianovasto-Chieti - Chieti

3. Comitato Etico Dell'a. O. Bianchi-Melacrino-Morelli - Reggio Calabria

4. Comitato Etico Dell'Irccs Istituto Nazionale Per Lo Studio E La Cura Dei Tumori Fondazione Giovanni Pascale - Naples

5. Comitato Etico Dell A. O. V. Monaldi - Naples

6. Comitato Etico Dell A. O. Antonio Cardarelli - Naples

7. Comitato Etico Indipendente Dell'A. O. U. Policlinico S. Orsola-Malpighi - Bologna

8. Comitato Etico Della Provincia Di Modena - Modena

9. Comitato Etico Unico Per La Provincia Di Parma - Parma

10. Comitato Etico Provinciale Di Reggio Emilia - Reggio Emilia

11. Comitato Etico Della Ausl Di Piacenza - Piacenza

12. Comitato Etico Di Area Vasta Romagna E Istituto Scientifico Romagnolo Per Lo Studio E La Cura Dei Tumori Di Meldola - Meldola

13. Comitato Etico Dell Azienda Policlinico Umberto I - Rome

14. Comitato Etico Indipendente Fondazione Ptv Policlinico Tor Vergata - Rome

15. Comitato Etico Dell'ausl Di Viterbo - Viterbo

16. Comitato Etico Dell'asl 3 Genovese - Genoa

17. Comitato Etico Dell'Ente Ospedaliero Ospedali Galliera - Genoa

18. Comitato Etico Dell A. O. Universitaria S. Martino - Genoa

19. Comitato Etico Fondazione S. Maugeri - Pavia

20. Comitato Etico Dell A.O. Ospedale Di Circolo - Busto Arsizio

21. Comitato Etico A.O. G. Salvini - Garbagnate Milanese

22. Comitato Etico Istituti Clinici Di Perfezionamento - Milan

23. Comitato Indipendente Di Etica Dell'a.O. Carlo Poma - Mantova

24. Comitato Etico Scientifico A. O. U. Policlinico G. Martino - Messina

25. Comitato Etico Interzonale Della Asur Zona Territoriale 8 Di Civitanova Marche E Zona Territoriale 9 - Macerata

26. Comitato Etico Dell'azienda Sanitaria Provinciale - Trapani

27. Comitato Etico Dell'a.O. U. S. Giovanni Battista - Torino

28. Comitato Etico Della Asl To/2 Di Torino - Gradenigo

29. Comitato Etico Dell A. O. U. Policlinico-Vittorio Emanuele - Catania

30. Comitato Etico Lazio 2 - Sora

31. Comitato Etico Dell'Irccs Multimedica - Sesto San Giovanni

32. Comitato Etico Interzonale Della Asur Zona Territoriale 8 Di Civitanova Marche E Zona Territoriale 9 - Macerata

33. Comitato Etico Asl Di Cagliari - Cagliari

34. Comitato Etico Di Bioetica Ospedale San Pietro - Roma

35. Comitato Etico Indipendente Istituto Nazionale Dei Tumori - Milano

36. Comitato Etico Fondazione S. Raffaele Del Monte Tabor - Milano

37. Comitato Etico A.O. S. Paolo - Milano

38. Comitato Etico Dell 'A. O. Della Valtellina E Della Valchiavenna - Morbegno

39. Comitato Etico Per La Sperimentazione Clinica - Mirano

40. Comitato Etico Milano Area B - Milan 


\section{Great study (NCT02293785) - 14 centers}

1. Comitato Etico Milano Area B - Milan (Coordinating Center)

2. Comitato Etico Dell"'Ao Specialistica Dei Colli" Monaldi-Cotugno-Cto - Naples

3. Comitato Etico Sapienza - Rome

4. Comitato Etico Per La Sperimentazione Clinica - Mirano

5. Comitato Etico Per Le Sperimentazioni Cliniche (Cesc) - Padua

6. Comitato Etico Azienda Usl Di Piacenza - Piacenza

7. Comitato Etico Ospedale San Raffaele - Milan

8. Comitato Etico Interaziendale Novara - Biella

9. Comitato Etico Indipendente Fondazione Ptv Policlinico Tor Vergata - Rome

10. Comitato Etico Regione Liguria - Genoa

11. Comitato Etico Indipendente, Cro Aviano - Aviano

12. Comitato Etico Palermo 2 - Trapani

13. Comitato Etico Lazio 1 - Rome

The Journal of Pain Research is an international, peer reviewed, open access, online journal that welcomes laboratory and clinical findings in the fields of pain research and the prevention and management of pain. Original research, reviews, symposium reports, hypothesis formation and commentaries are all considered for publication

\section{Dovepress}

The manuscript management system is completely online and includes a very quick and fair peer-review system, which is all easy to use. Visit http://www.dovepress.com/testimonials.php to read real quotes from published authors. 